\title{
Pathogenese und Pathophysiologie der Narkolepsie
}

\author{
Geert Mayer und Thomas Pollmächer
}

\begin{abstract}
Zusammenfassung
Die Narkolepsie besticht durch die Vielfältigkeit ihrer Symptome. Durch die Entdekkung des Neuropeptids Orexin, das im Liquor von Narkolepsiepatienten nicht nachweisbar ist, konnten neue Einblicke in die Pathophysiologie der Erkrankung gewonnen werden. Die Orexine beeinflussen verschiedene an der Schlafregulation beteiligte Neurotransmittersysteme. Der Orexinmangel kann sowohl eine verminderte Exzitation noradrenerger Neurone als auch eine Enthemmung des cholinergen Tonus verursachen. Durch diese Veränderung des Zusammenspiels lassen sich einige narkoleptische Symptome einfach erklären. Narkolepsieähnliche Symptome können im Tierversuch durch Manipulation des Orexinsystems erzeugt werden. Als Erkrankung mit der stärksten HLA-Assoziation beflügelt konsequenterweise die bisher unbeantwortete Frage, ob die Narkolepsie durch einen Autoimmunprozess orexinhaltige Neurone zerstört, den wissenschaftlichen Forschungsprozess.
\end{abstract}

\begin{abstract}
Pathogenesis and pathophysiology of narcolepsy.

The sleep disorder narcolepsy impresses by its variety of symptoms. With the discovery of the neuropeptide orexin, which is not detectable in the CSF of narcoleptic patients, new insights into the pathophysiology of the disorder were gained. Orexin exerts influence on different neurotransmitter systems that are involved in sleep regulation. The deficit in orexins can cause decreased excitation of noradrenergic neurons in the locus coeruleus and vice versa disinhibition of cholinergic tone, thus explaining many narcoleptic symptoms. Narcolepsy like symptoms can be produced in animals by experimentally induced manipulations within the orexin system. In this disorder which has the highest known association with HLA, the intriguing question if narcolepsy may be caused by an autoimmune process that destroys neurons containing orexin, pinpoints future scientific effort.
\end{abstract}

Key words: narcolepsy; NREM-, REM-sleep; neurotransmitter imbalance; orexins

\section{Einleitung}

Seit ihrer Entdeckung vor mehr als hundert Jahren fasziniert die Narkolepsie durch die Vielfältigkeit und Prägnanz ihrer Symptome Kliniker und Wissenschaftler. Die seltene Erkrankung betrifft Tier und Mensch. Es existieren Hundemodelle (Mignot et al. 1992) mit autosomal rezessivem Erbgang und eine Reihe von transgenen Mausmutanten, die typische Symptome der Erkrankung zeigen (Chemelli et al. 1999). Für die humane Narkolepsie ist eine familiäre Häufung seit langem bekannt, der Beitrag der Genetik ist aber wesentlich komplexer als im Tiermodell und der Vererbungsmodus ist multifaktoriell.

Die Entdeckung der Assoziation der Narkolepsie mit einem humanen Leukozytenantigen, dem HLA DR2, die mit 98,5\% die engste ist, die je für eine Erkrankung beschrieben wurde, hat in den 1980er Jahren zu der Hypothese geführt, dass die Narkolepsie eine Autoimmun-Erkrankung ist (Honda et al.1986). Bislang konnten allerdings keine konsistenten Hinweise für eine kausale Beurteilung immunologischer Faktoren gefunden werden (Hinze-Selch et al. 1998), wobei stets vermutet wurde, dass immunologische Auffälligkeiten bei Narkolepsiepatienten wegen einer engen zeitlichen und räumlichen Begrenzung autoimmunologischer Phänomene schwer fassbar sein könnten.

Die Entdeckung, dass bei Narkolepsiepatienten Orexin-A, ein erst kürzlich entdecktes Neuropeptid, im Gegensatz zu Gesunden im Liquor nicht nachweisbar ist (Nishino et al. 2000; Dalal et al. 2001), hat autoimmunologischen Hypothesen zur Narkolepsieentstehung neue Nahrung gegeben, zumal zusätzlich gezeigt werden konnte, dass die Zahl orexin-reaktiver Neurone im lateralen Hypothalamus deutlich vermindert ist (Peyron et al. 1998; Thannickal et al. 2000).

\section{Das Klinische Bild der Narkolepsie}

Die Narkolepsie ist eine lebenslang andauernde Schlafstörung unbekannten $\mathrm{Ur}$ sprungs. Etwa 0,05\% der Bevölkerung sind davon betroffen. Die Hauptsymptome sind eine vermehrte Tagesschläfrigkeit und Kataplexien. Kataplexien stellen einen plötzlich einsetzenden und nur kurz (Sekunden bis wenige Minuten) anhaltenden partiellen oder kompletten Verlust des Tonus der Halte- und Stellmuskulatur dar, der durch Emotionen, insbesondere durch Lachen und freudige Erregung, ausgelöst wird.

Die Tagesschläfrigkeit fluktuiert im Verlaufe des Tages. Im Tagsschlaf können sowohl REM- als auch NREM-Schlafepisoden auftreten, wobei REM-Schlafepisoden klinisch oft mit sehr lebhaften Träumen und Muskelatonie einhergehen. Für Patienten mit Narkolepsie sind nicht nur sehr rasche Übergänge zwischen Schlafstadien typisch, sondern auch Manifestationen einzelner Aspekte des NonREM und REM-Schlafs, selbständig und unabhängig vom Schlaf selbst im Sinne einer dissoziativen Manifestation einzelner Schlafstadien. Hierdurch entstehen ungewöhnliche Symptomkonstellationen: Bei REM-Schlaf-Einbrüchen in den Wachzustand wird die reale Umgebung zusammen mit dem Trauminhalt wahrgenommen (hypnagoge Halluzination) und es kann zu dem für den REM-Schlaf typischen Tonusverlust der Haltemuskulatur kommen, der als Schlaflähmung bezeichnet und meist als sehr beängstigend erlebt wird. Bei sehr starken Gefühlen, am häufigsten bei Freude, Lachen oder Überraschung, tritt die schon erwähnte Kataplexie auf, bei der - ähnlich wie im REM-Schlaf - aus dem Wachzustand heraus ein Tonusverlust der Halte- und Stellmuskulatur auftritt, der von unterschiedlicher Intensität sein kann und selektiv z.B. nur die mimische Muskulatur, aber auch die gesamte Muskulatur betreffen kann. Fakultative, unspezifische Symptome sind hypnagoge Halluzinationen, automatisches Verhalten, Schlaflähmungen und ein gestörter Nachtschlaf. Die Kataplexie ist als einziges Symptom pathognomonisch für die Narkolepsie.

Die Kernsymptome der Narkolepsie entwickeln sich meist ungleichzeitig und können sich im Mittel mit einem Abstand von bis zu 10 Jahren manifestieren. Meist beginnt die Erkrankung mit dem Symptom Tagesschläfrigkeit. Der Erkrankungsbeginn weist zwei Häufigkeitsgipfel zwischen dem 10.20. und dem 30.- 40. Lebensjahr auf (Dauvilliers et al. 2001; Mayer et al. 2002). Da das Symptom Tagesschläfrigkeit unspezifisch ist, wird die Diagnose meist erst nach 


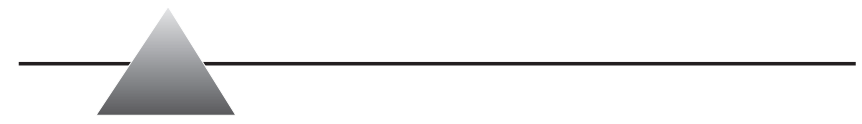

Manifestation des spezifischen Symptoms Kataplexie gestellt. Die Zeitspanne zwischen den beiden Symptomen ist bei Erstmanifestationen der Tageschläfrigkeit im zweiten Lebensjahrzehnt länger als bei Erstmanifestationen im vierten Lebensjahrzehnt.

Die Narkolepsie weist eine erhöhte Komorbidität mit Parasomnien auf, die selbst keine Schlaf-Wachstörungen sind, sondern als unerwünschte motorische Ereignisse im Schlaf klassifiziert sind. In einer eigenen retrospektiven Untersuchung bei Narkolepsiepatienten (Mayer et al. 2002) fanden sich bei $14,2 \%$ der Patienten die NREM-Parasomnie Schlafwandeln $(2,5 \%$ in der erwachsenen Bevölkerung), die REM-Parasomnien Albträume bei 41,5\% (5-7\% in der Bevölkerung ) und Verhaltensstörungen im REM-Schlaf bei $18,9 \%$ ( $0,5 \%$ in der Bevölkerung).

Die Patienten leiden unter der schläfrigkeitsbedingten Leistungsminderung, ohne dass die geistigen oder körperlichen Leistungen gegenüber Gesunden tatsächlich vermindert sind. Die Fähigkeit, kognitive Dauerleistungen zu vollbringen ist allerdings beeinträchtigt. Gegenüber Gesunden ist die geteilte und fokussierte Aufmerksamkeit herabgesetzt (Rieger et al. 2003).

\section{Genetik}

Unterschiedliche Untersuchungen der letzten 50 Jahre haben wegen unscharfer Operationalisierung der Narkolepsie eine sehr variable und wahrscheinlich meist zu hohe familiäre Häufung zwischen 5$52 \%$ festgestellt. Unter Berücksichtigung des Symptoms Kataplexie reduziert sich das Risiko für einen Verwandten ersten Grades auf ca. 1-2\% (Overeem et al. 2001). Dieses Risiko ist aber immer noch 30- 40 mal höher als die Prävalenz in der Bevölkerung, was die wichtige Rolle genetischer Faktoren in der Pathogenese eindrücklich unterstreicht. Die Narkolepsie zeigt von allen bekannten Erkrankungen die höchste Assoziation mit dem humanen Leukozyten Antigen HLA-DR2 (85-95\%, Nishino et al. 2000). Die enge Assoziation lässt, wie schon erwähnt, eine Beteiligung des Immunsystems an der Pathogenese vermuten. Dieses Antigen wird bei Berücksichtigung aller ethnischen Gruppen aber nur bei $62 \%$ gefunden, das Allel HLA DQB1*0602 hingegen wieder bei ca. 85\% (Mignot et al. 2001). Die Assoziation zum DQB $1 * 0602$ Antigen ist nur sehr hoch bei Patienten mit typischen Kataplexien.

Beide Gene liegen auf Chromosom 6 eng benachbart. Gesunde, von denen ca. $25 \%$ HLA DQB1*0602 positiv sind, haben eine kürzere REM-Latenz als Gesunde ohne das Allel (Mignot et al. 1998). Aus diesen Befunden wird abgeleitet, dass HLA DQB1*0602 ein Suszeptibilitäts-Gen für Narkolepsie ist.

Untersuchungen an Familien mit mehreren Narkolepsiepatienten oder konkordanten eineiigen Zwillingen deuten allerdings darauf hin, dass gerade in Familien mit mehreren Patienten die Erkrankung häufig unabhängig vom typischen HLA-DQB1*0602 vererbt wird. In diesen Fällen findet sich bei HLA DQB1*0602 positiven Patienten, deren Eltern nicht über das Allel verfügen, gehäuft zusätzlich HLA DB1*0301, dem damit möglicherweise ebenfalls die Rolle eines Suszeptibilitätsgens zukommt (Mignot et al. 2003). Da auch in den Familien mit mehreren Betroffenen kein eindeutiger Erbgang festgestellt werden konnte, muss von einer multifaktoriellen Genese ausgegangen werden. Dies bedeutet, dass eines oder evtl. auch mehrere Gene zwar eine erhebliche Suszeptibilität für das Entstehen der Erkrankung bedingen, dass aber zusätzlich nicht-genetische Faktoren eine große Rolle spielen. Zu diesem pathogenetischen Modell passt sehr gut die Entdeckung, dass beim Menschen die Narkolepsie mit einer erworbenen Defizienz des Orexinsystems einhergeht, die im folgenden näher beschrieben ist.

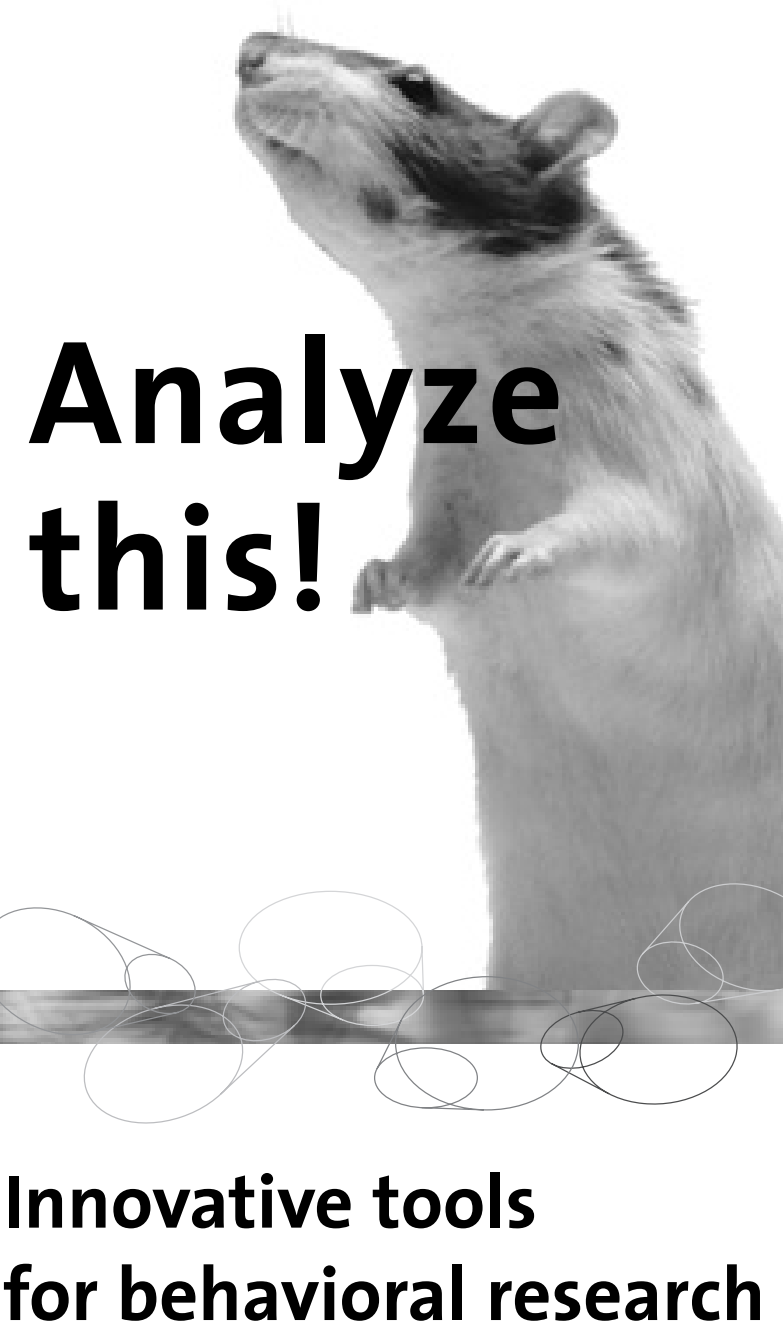

for behavioral research

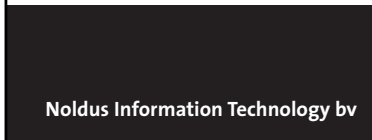

Wageningen, The Netherlands

Phone: $\quad+31-317-497677$

E-mail: info@noldus.nl

Noldus Information Technology GmbH

Freiburg, Germany

Phone: $\quad+49-761-4701600$

E-mail: info@noldus.de

Noldus Information Technology Inc.

Leesburg, VA, U.S.A.

Phone: $\quad+1-703-771-0440$

Toll-free: 1-800-355-9541

E-mail: info@noldus.com

www.noldus.com

\section{Scientists studying animal behavior}

have an increasing need for accurate

quantitative data. As a behavioral neuro-

scientist, you need sensitive observational

research tools with a maximum degree of automation. Our integrated solutions for data collection, analysis, management and visualization are today's premier tools for the study of behavior, locomotion and acoustics.

EthoVision - Video tracking system for automation of behavioral experiments The Observer - System for collection and analysis of observational data, live or from video

UltraVox - System for automatic monitoring of ultrasonic vocalizations 


\section{Pathophysiologie}

Die Rolle der Orexine in der Narkolepsie. 1999 wurden erste Untersuchungen publiziert, die auf eine zentrale pathophysiologische Rolle eines neu entdeckten Neuropeptidsystems in der Pathophysiologie der Narkolepsie hinweisen (Review siehe Hungs und Mignot 2001; Chemelli et al. 1999; Lin et al. 1999). Orexine, auch Hypocretine genannt, sind wahrscheinlich fast ausschließlich im lateralen Hypothalamus produzierte Neuropeptide mit einem gemeinsamen Präkursorpeptid. Aus diesem Präproorexin wird sowohl Orexin-A als auch Orexin-B (Hypocretin-1 und Hypocretin-2) gebildet (Sakurai et al. 1999). Orexin-Neuronen projizieren in eine große Zahl von Gehirnregionen einschließlich verschiedener Hirnstammregionen wie dem Locus Coeruleus und kortikaler Areale wie dem präfrontalen Kortex (Peyron et al. 1998) (Abbildung 1).

lichen Symptomen (Hara et al. 2001). Charakteristisch sind hierfür bei der Maus spontan oder aber auch im Rahmen sozialer Interaktion auftretende Episoden motorischer Inaktivität, die der Kataplexie beim Menschen ähnelt.

Im Liquor von Narkolepsiepatienten sind die Orexin-A-Spiegel im Vergleich zu denen gesunder Kontrollpersonen typischerweise stark reduziert; bei den meisten Patienten (> 90\%) kann Orexin-A überhaupt nicht nachgewiesen werden (Nishino et al. 2000; Dalal et al. 2001).

Es ist bisher noch unklar, was pathophysiologisch Patienten mit und ohne messbare Orexin-A-Spiegel im Liquor unterscheidet. Es gibt bisher keine Hinweise darauf, dass Symptomatik oder Verlauf unterschiedlich wären, allerdings weisen auffällig viele, wenn auch nicht alle Patienten mit messbaren Orexinspiegeln (Dalal et al. 2002; Kanbayashi et al. 2002) nicht das narkolepsie-

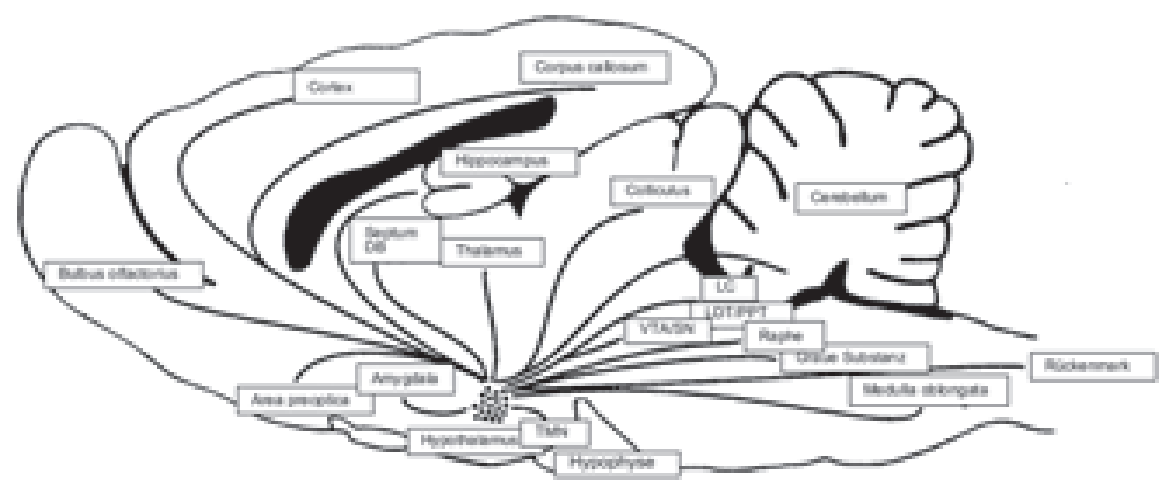

Abb. 1: Hypocretinhaltige Neurone und ihre Projektionen innerhalb des Gehirns Schwarze Punkte: Orexinhaltige Neurone des Hypothalamus mit LC: Locus Coeruleus, LDT/PPT: Laterodorsales Tegmentum/Pedunkulopontines Tegmentum, TMN: Tuberomamillärer Nukleus, VTA/SN: Area ventralis tegmentalis/ Substanzia nigra

Ihre Wirkung entfalten Orexine über zwei Rezeptoren (ORX-1 und ORX-2, bzw. Hcrt1 und Hcrt-2 Rezeptor), mit teilweise differentieller Affinität für die beiden Peptide.

Mehrere Studien zeigten, dass ein Mangel an Orexinen oder eine defekte Signalübertragung im Hypothalamus bei Tieren eng mit narkolepsie-artigen Symptomen verbunden ist: Narkolepsie bei Hunden wird durch einen genetischen Defekt im ORX-2Rezeptor-Gen bewirkt (Lin et al. 1999); darüber hinaus zeigen Mäuse mit einem defekten Präproorexin-Gen einen narkolepsieähnlichen Phänotyp (Chemelli et al. 1999).

Schließlich führt die Kopplung des Orexin-Gens mit dem Ataxin-3-Gen bei Mäusen zu einem Untergang orexin-produzierender Zellen im Laufe der post-natalen Entwicklung und ebenfalls zu narkolepsie-ähn- typische HLA-Antigen auf. Während also normale Orexin-A-Konzentrationen im Liquor die Diagnose einer Narkolepsie nicht ausschließen, scheint umgekehrt das Fehlen des Peptids hochspezifisch für die Erkrankung zu sein, denn abgesehen von einigen wenigen Patienten mit Guillain-Barré-Syndrom konnte bisher keine andere Erkrankung identifiziert werden, bei der im Liquor verminderte Orexin-Konzentrationen auftreten. Hingegen wurde kürzlich beim Restless Legs-Syndrom (Allen et al. 2002) über leicht erhöhte Orexin-A-Konzentrationen im Liquor berichtet, allerdings bei einer kleinen Zahl vorbehandelter Patienten, so dass dieser Befund zunächst einmal der Replikation bedarf.

In zwei unabhängigen Studien an postmortem entnommenen Hirngewebe von Narko- lepsiepatienten fand sich eine deutlich verringerte Anzahl orexinhaltiger Neuronen im Hypothalamus (Peyron et al. 2000; Thannickal et al. 2000). Eine dieser Studien beschrieb darüber hinaus eine Gliose in diesem Bereich, was darauf hindeutet, dass eine Schädigung dieser Zellen bei Einsetzen der Erkrankung einen entscheidenden Schritt in der Pathophysiologie der Narkolepsie darstellen könnte. Dies lässt vermuten, dass ein im Rahmen der Narkolepsie beim Menschen auftretender Orexinmangel erworben und nicht durch einen genetischen Defekt im System bedingt ist. Diese Annahme wird auch durch Ergebnisse genetischer Untersuchungen gestützt, die, abgesehen von einem einzigen Fall einer Narkolepsie im Säuglingsalter, keine Defekte des PräproorexinGens oder der Orexinrezeptorgene feststellen konnte (Gencik et al. 2001; Peyron et al. 2000).

Derzeit wird diskutiert, ob Orexine auch außerhalb des ZNS gebildet werden und ob die Messung von Orexin-Konzentrationen im peripheren Blut als diagnostischer Parameter aussichtsreich ist (Dalal et al. 2002). Die Befunde sind bei Narkolepsiepatienten und Gesunden sehr widersprüchlich (Dalal et al. 2001, 2002; Higuchi et al. 2002; Adam et al. 2002; Nishino et al. 2001; Arihara et al. 2001; Komaki et al. 2001), so dass bisher ausschließlich die Messung von Orexinen im Liquor als klinisch relevanter Parameter betrachtet werden kann.

Sicher ist derzeit, dass die Narkolepsie beim größten Teil der Patienten einen HLAassoziierten genetischen Hintergrund hat. Auf diesem Hintergrund und aus bisher unbekannter Ursache erfolgt entweder eine Zerstörung orexin-produzierender Neuronen oder aber diese Neuronen stellen die Orexinproduktion ein. Die Suche nach neuronenspezifischen und organ-spezifischen Autoantikörpern im Serum konnten die Hypothese, dass Narkolepsie eine Autoimmunerkrankung ist, bisher nicht erhärten (Black et al. 2002).

Was auch immer die detaillierte Ursache des Orexinmangels ist, er beeinträchtigt verschiedene an der Schlafregulation beteiligte Neurotransmittersysteme. Die bisher ungeklärte Frage ist, wie das Orexinsystem mit cholinergen und monoaminergen Neuronen in Wechselwirkung tritt, so dass Symptome der Narkolepsie auftreten (Abbildung 2).

In Hundemodellen wurde in der Formatio reticularis und im basalen Vorderhirn eine cholinerge Überempfindlichkeit beschrieben, die letztendlich zu den im Rahmen der Narkolepsie auftretenden REM-Schlaf-Anomalien beitragen könnte (Nishino et al. 

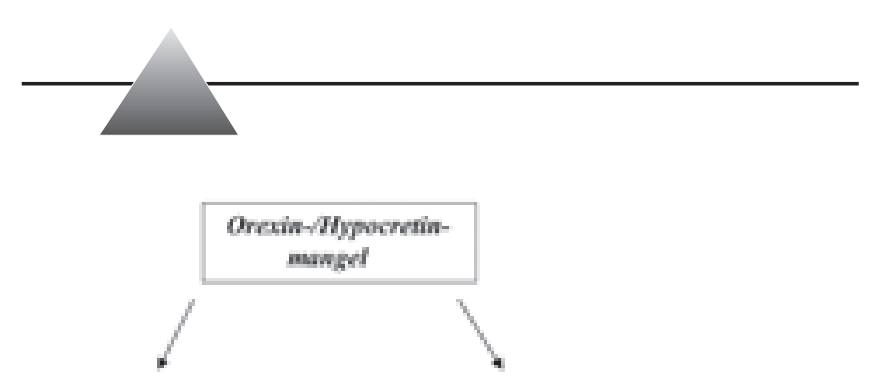

Chowinerge

Hyperaknivinar

,

\section{Symplame:}

Katioploile

Hopnogoge Halherinatioesn

S-hlaphishereg

Pobsomnographische Refunde: Shoponed-REM-Epiodos

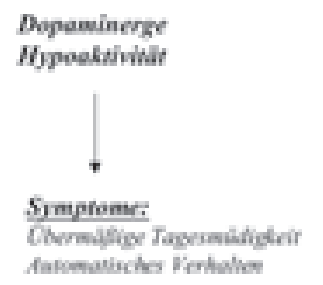

Pofrommegrophische Refounde: Verkìrale Eiechloflative

Abb. 2: Auswirkungen des Orexin-/Hypocretinmangels auf Neurotransmitter, Symptome und polysomnographische Befunde

1997). Orexinneurone im Hypothalamus projizieren in die verschiedensten Hirnregionen, am stärksten jedoch in den Locus Coerulus, wo sie eine stark exzitatorische Wirkung auf die dort befindlichen noradrenergen Neuronen ausüben. Im Falle eines Orexinmangels sollte es deshalb während des REM-Schlafes zu einer reduzierten Feuerrate des Locus Coerulus kommen, was eine REM-Schlaf-Disinhibition zur Folge hätte (Kilduff et al. 2000), siehe Abbildung 1.

Die ausgedehnten Projektionen des orexinergen Systems in subkortikale und kortikale Areale deutet darauf hin, dass sich ein Versiegen der Orexinproduktion auf weite Teile des Gehirns auswirken kann. Dies wird auch durch eine jüngst erschienene Arbeit zur grauen Substanz bei Narkolepsie/Kataplexie-Patienten im Vergleich zu gesunden Probanden gestützt (Kaufmann et al. 2002). Bei den Patienten wurde eine deutliche bilaterale, kortikale Reduktion der grauen Substanz vor allem in den temporobasalen und frontobasalen Arealen beobachtet, was in einer globalen Reduktion des Volumens der grauen Substanz resultiert. Es ist unklar, ob dieser kortikale Zellverlust aufgrund einer umfassenderen primären Pathologie (z.B. einem autoimmun vermittelten Zellverlust) oder sekundär als Folge fehlenden Inputs durch Orexin enthaltende Neuronen entsteht.

Die Einbindung der Orexine in hypothalamische Netzwerke, die Appetit, Gewicht und Metabolismus regulieren, lässt vermuten, dass auch diese ZNS-Funktionen bei Narkolepsiepatienten verändert sind. Die initiale Entdeckung, dass Orexine appetitstimulierend wirken, führte zunächst zur Hypothese, dass Narkolepsiepatienten verminderten Appetit und ein vermindertes Körpergewicht zeigen sollten. Interessanterweise ist dies aber nicht der Fall. Im Gegenteil, schon seit den 30er Jahren wurde anekdotisch berichtet, dass Narkolepsiepatienten sehr häufig übergewichtig sind (Daniels 1934). Dieser Befund konnte kürzlich im Vergleich zu einer großen Kontrollpopulation statistisch gesichert (Schuld et al. 2000) und auch unabhängig repliziert werden (Dahmen et al. 2001). Zu Nahrungsaufnahme und Appetit bei Narkolepsiepatienten ist bisher leider sehr wenig bekannt. Während schon in den 70er Jahren über Heißhunger auf Süßigkeiten und vermehrten Appetit berichtet wird (Bell 1976), (ein Befund, der durch die Ergebnisse im Max-Planck-Institut für Psychiatrie durchgeführten, noch nicht veröffentlichten Befragung von Patienten gestützt wird), berichtet eine neuere Studie über eine verminderte Nahrungsaufnahme (Lammers et al. 1996). Allerdings wurde diese Untersuchung an langjährig Erkrankten durchgeführt und die Erhebung bezog sich auf die aktuellen Verhältnisse. Deshalb ist es denk-

Neuroforum 4/03

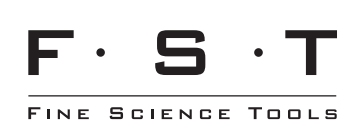

Fine surgical instruments and accessories for research

- Spring scissors

- Forceps

- Scalpels

- Sutures

- Retractors

- Clamps

- And much more

Fine Science Tools GmbH

Fahrtgasse 7 - 13

D-69117 Heidelberg

Germany

Tel.: +49 (0) $6221 / 905050$

Fax: +49 (0) $6221 / 600001$

E-Mail: europe@finescience.com Web: www.finescience.com

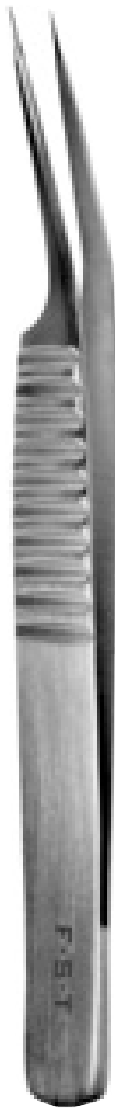

\section{Glaskapillaren zur Herstellung von}

\section{Mikroelektroden, Mikropipetten, Patch-Pipetten, etc.}

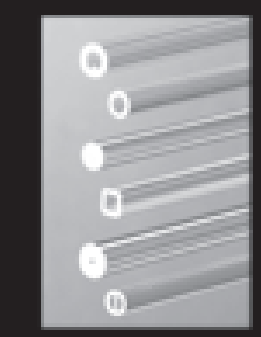

\section{Direkt vom Hersteller:

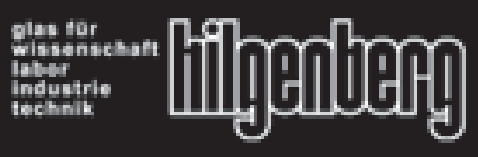

In allen Längen lieferbar...

auch mit feurpolierten Enden ...

... aus Borosilicat-, Soda-, Quarz- und Bleiglas

Verschiedene Formen:

- rund, eckig

- multibarrel

- Theta , Filament

- 2-Loch, 4-Loch

- usw.

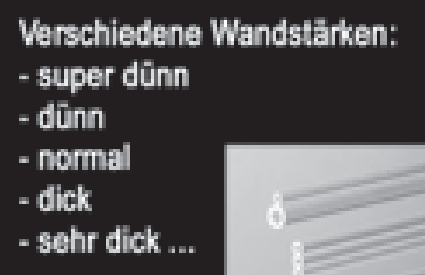

Neu:

Spezialnadein ab $70 \mu \mathrm{m}$ Durchmesser, zum Befüllen von Mikroelektroden und Mikropipetten!

Hilgenberg GmbH, Strauchgraben 2, D - 34323 Malsfeld

Tel. ++49 (0) 56617303 o Fax ++49 (0) 5661730311

Email info Qhilgenberg-gmbh.de Internet www.hilgenberg-gmbh.de 


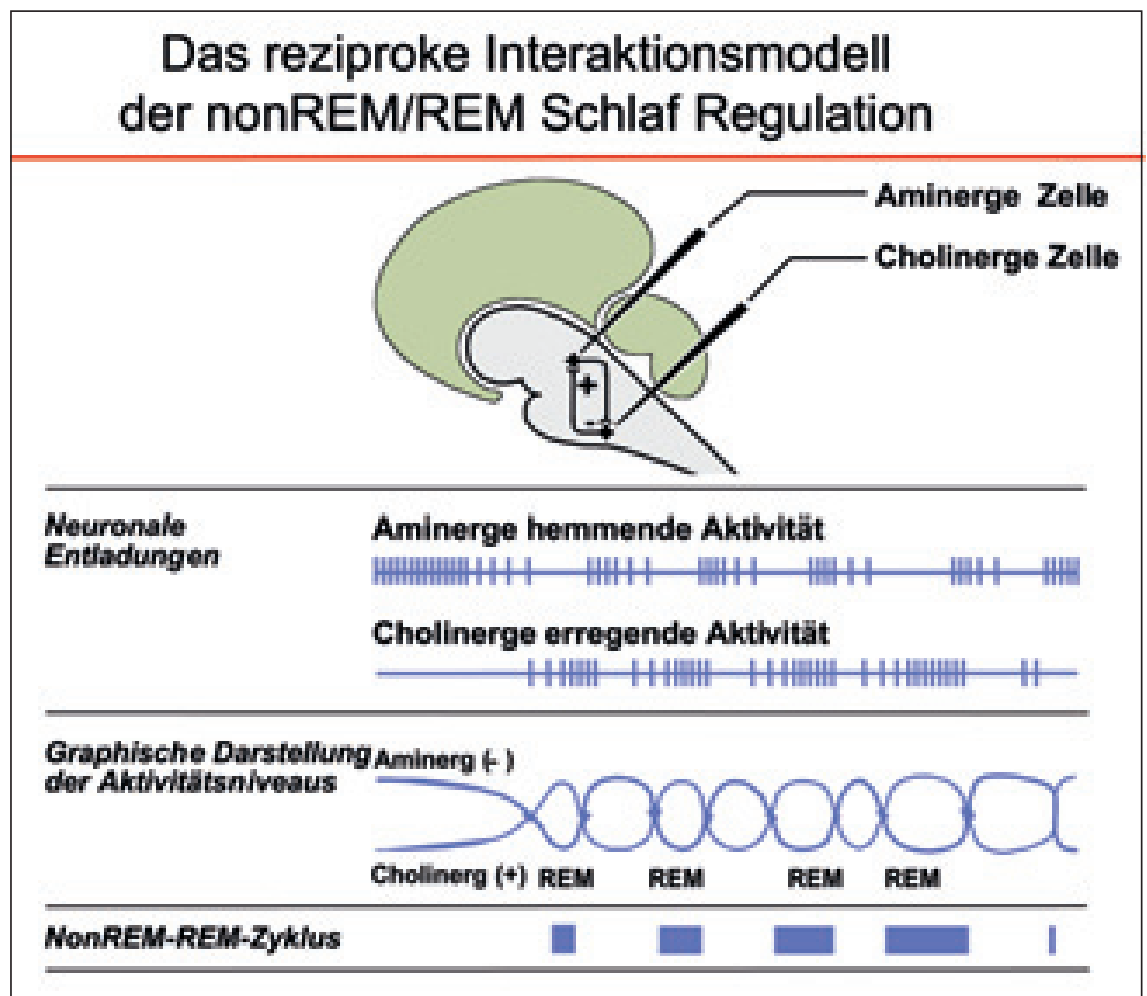

Abb. 3: Das reziproke Interaktionsmodell der nonREM/REM Schlaf Regulation

bar, dass eine verminderte Nahrungsaufnahme die bewusste Konsequenz ist, die Patienten aus dem Narkolepsie-assoziierten Übergewicht ziehen.

Die Tatsache, dass Patienten mit Narkolepsie trotz des Fehlens eines orexigenen Peptides häufig übergewichtig sind, ist nur auf den ersten Blick paradox. Zum einen zeigt eine Reihe von Studien, dass chronische Applikation von Orexinen nicht zu Übergewicht führt. Hierzu passend sind Präproorexin-Knockout-Mäuse nicht adipös (und auch nicht untergewichtig). Transgene Mäuse allerdings, bei denen es in der nachgeburtlichen Entwicklung zu einer Zerstörung orexin-produzierender Zellen kommt (Orexin/Ataxin-3-Mäuse, Hara et al. 2001) zeigen sehr wohl eine relativ spät einsetzende Adipositas. Dieses transgene Modell entspricht wahrscheinlich eher der pathophysiologischen Situation bei der humanen Narkolepsie, da es, wie oben ausgeführt, wahrscheinlich ist, dass die orexin-produzierenden Zellen erst zu Beginn der Erkrankung zugrunde gehen. Es liegt also die Vermutung nahe, dass im Tiermodell und bei der humanen Narkolepsie nicht der Orexinmangel, sondern andere Faktoren, die durch den funktionellen Ausfall der entsprechenden Zellen bedingt sind, eine Gewichtszunahme induzieren.
Die Rolle von Leptin in der Narkolepsie. Der erhöhte BMI bei Narkolepsiepatienten kann also wahrscheinlich nicht ausschließlich mit einem Orexinmangel im Hypothalamus erklärt werden. Vermutlich ist eine wesentlich komplexere Störung der Appetitregulation und des Essverhaltens für dieses Phänomen verantwortlich. Aus Studien an Mäusen, die infolge genetischer Defekte im Leptinsystem extrem übergewichtig waren, ist bekannt, dass diese Tiere auch unter einem zentralen Orexinmangel leiden (Yamamoto et al. 1999). Bei Leptin handelt es sich um eines der wichtigsten Sättigungssignale der peripheren Fettspeicher an die appetitregulierenden Zentren im Gehirn (Blum et al. 1997). Es spielt daher eine wichtige Rolle bei der Regulation des Essverhaltens im Hypothalamus (Schwartz et al. 2000). Die Konzentrationen dieses Hormons im Blut und Liquor einer Gruppe von Narkolepsiepatienten und zweier Kontrollgruppen, die bezüglich Geschlechtsverteilung, mittleren Alters und BMI parallelisiert waren (eine Patientengruppe litt unter Depressionen, die andere unter nicht-entzündlichen neurologischen Erkrankungen) wurden am Max-Planck-Institut München bestimmt (Schuld et al. 2000). Die Leptinspiegel im Liquor unterschieden sich zwar in den einzelnen Gruppen nicht signifikant, doch die Serumleptinspiegel lagen bei Narkolepsiepatienten signifikant niedriger als bei den Kontrollgruppen. Dies deutet darauf hin, dass das Gehirn der Patienten über die Lage der peripheren Fettspeicher falsch informiert ist, was die erhöhte Nahrungsaufnahme erklären könnte. Eine weitere unabhängige Studie bestätigte die Senkung des Plasmaleptinspiegels und belegte außerdem eine gedämpfte zirkadiane Rhythmik bei Narkolepsiepatienten (Kok et al. 2002). Die Plasmaleptinspiegel waren erniedrigt, wohingegen der nächtliche Leptin-Peak einer normalen zirkadianen Leptinrhythmik fehlte. Eine andere Arbeitsgruppe hat einen im Vergleich zu Kontrollpersonen signifikanten Anstieg des Leptins im Liquor von Narkolepsiepatienten beschrieben, was darauf hindeutet, dass ein erhöhter Leptinspiegel im Liquor eine Begleiterscheinung eines erhöhten BMI sein kann. In der Prospektivstudie wurde jedoch der Unterschied bezüglich des BMI bei Narkolepsiepatienten und Kontrollpersonen sowie bezüglich des Geschlechts nicht kontrolliert (Nishino et al. 2001).

Die Rolle von Prostaglandin in der Narkolepsie. Ob Orexine allein für die Regulation von Kataplexien, Tagesschläfrigkeit und die anderen Narkolepsiesymptome kausal verantwortlich sind, ist nicht geklärt. Im Tiermodell hat das Prostaglandin-D-System eine bedeutende Rolle in der Schlaf-WachRegulation, die sich auf Primaten übertragen ließ (Urade et al. 1999). Auch bei schlafgesunden Menschen ist die Prostaglandin-D-Synthase (PGDS) an der Schlafregulation beteiligt (Jordan et al. 2000, 2002). Die Veränderung des ProstaglandinD-Systems bei Menschen mit Erkrankungen, deren Leitsymptom Hypersomnie ist (Afrikanische Schlafkrankheit, systemische Mastocytose, bakterielle Meningitis) ist gut belegt. Untersuchungen bei Hunden (Nishino et al. 1989) zeigen den Einfluss von PGDS auf das Symptom Tagesschläfrigkeit, nicht jedoch Kataplexie. In gemeinsamen Untersuchungen der Psychiatrischen Universitätsklinik/Göttingen und der HephataKlinik/Treysa an Narkolepsiepatienten wurde in regelmäßigen Intervallen die Konzentration von PGDS im Serum, Speichel und fraktioniertem Urin über eine Periode von fünf Tagen und Nächten, in der physiologischer Schlaf, REM-Schlafentzug und vollständiger Schlafentzug eingeschlossen waren, gemessen. Die PGDS-Serumkonzentration bei Narkolepsiepatienten war gegenüber der von Kontrollpersonen signifikant erhöht, ohne erkennbare Beeinflussung durch kompletten oder REM-Schlafentzug 
zu zeigen. Bei einem Patienten mit idiopathischer Hypersomnie fanden sich ebenfalls erhöhte PGDS-Werte, so dass angenommen werden kann, dass PGDS für das Symptom Tagesschläfrigkeit krankheitsunspezifisch verantwortlich sein kann.

Die Rolle der Neurotransmitter in der Narkolepsie. Richtungsweisende Entdekkungen der letzten Jahrzehnte erklären den Schlaf-Wach- Rhythmus durch reziproke inhibitorische Interaktionen zwischen cholinergen (laterodorsales Tegment, pedunkulopontine Nuclei) und monoaminergen (Locus coeruleus, Raphe und tuberomamilläre Nuclei) Zellgruppen des Hirnstamms (Abbildung 3). Der monoaminerge Tonus ist während des Wachseins hoch und nimmt im Verlauf des Schlafzyklus ab. Die aszendierenden monoaminergen und cholinergen Projektionen steuern die EEG Veränderungen, die deszendierenden cholinozeptiven Neurone des Nucleus retikularis pontis projezieren zur Medulla oblongata und von dort zu den motorischen Vorderhornzellen, die sie über glyzinerge Interneurone hemmen und dadurch die Muskelatonie im REM-Schlaf herstellen. Eine Verminderung des monoaminergen Tonus führt zu einer Enthemmung cholinerger Neurone, die REM-Schlaf verursachen. Die Narkolepsie wird vermutlich durch ein Überwiegen des cholinergen Systems verursacht (Hungs und Mignot 2001).
Dopaminerge Mechanismen bei der Auslösung von Kataplexien (z.B. durch Gabe von Prazosin), die Wirksamkeit dopaminerger Stimulanzien und Ergebnisse postmortaler neuroanatomischer Untersuchungen (Aldrich 1992; Kish et al. 1992) einer vermehrten Dopamin $\mathrm{D}_{2}$-Rezeptorenbindung im Nucleus caudatus, Globus pallidus und den Amygdala, führten zu intensiver Erforschung des dopaminergen Systems. Rezeptorbindungsstudien im SPECT und PET (Kahn et al. 1994; Staedt et al.1998) zeigten jedoch eine normale $\mathrm{D}_{2}$ - und $\mathrm{D}_{1}$-Rezeptorendichte im Striatum von Narkolepsiepa-tienten. Auch bei einem diskordanten Zwillingspaar mit Narkolepsie fand sich beim CoZwilling eine normale $\mathrm{D}_{2}$ Rezeptorenbindung. Durch Blockade der $\alpha_{2} / \mathrm{D}_{2} / \mathrm{D}_{3}$ Rezeptoren und durch Stimulation von $\alpha_{1(\mathrm{~b})}$ Rezeptoren können Kataplexien verhindert werden, so dass Dopamin eine wichtige Rolle für die Pathophysiologie der Narkolepsie spielen muss.

In pharmakologischen Tierexperimenten (Lin et al. 1996; Engber et al. 1998) induzierten Amphetamininjektionen Fos-Immunoreaktivität in Neuronen des Striatum und Kortex, insbesondere im Nukleus Caudatus und mediofrontalem Kortex, Injektionen mit Modafinil induzierten Fos-Immunoreaktivität im anterioren Hypothalamus und Nucleus Caudatus. Keine Substanz markierte die primären Schlaf-Wachzentren. Die Immun- oreaktivität des Striatums auf Amphetamin ist mit deren Funktion, dopaminerge Übertragung zu verstärken, vereinbar. Der Effekt von Modafinil auf den Zentralkern der Amygdala verweist auf einen anderen stimulierenden Effekt wie Modulation von behavioralem und alertisierendem Arousal.

Tierstudien haben gezeigt, dass die lokale Injektion oder Perfusion cholinerger Agonisten in die zuvor genannten Areale REMSchlaf und Muskelatonie hervorrufen und so narkolepsie-ähnliche Symptome auslösen kann. Darüber hinaus wurde eine Hypoaktivität der mesokortikolimbischen dopaminergen Systeme im ventralen Tegmentum und der Substantia nigra belegt, die bei der Modulation von Kataplexie und Tagesmüdigkeit ebenfalls eine entscheidende Rolle zu spielen scheinen (Nishino und Mignot 1997).

\section{Therapie der narkoleptischen Symptome}

Therapeutisch lassen sich die narkoleptischen Symptome durch Anwendung von spezifischen Bewältigungsstrategien beeinflussen, aber nicht grundlegend ändern. Die Therapien sind ausgerichtet auf die Hauptsymptome. Kataplexien, Schlaflähmungen und hypnagoge Halluzinationen lassen sich unspezifisch mit trizyklischen und neueren Antidepressiva behandeln (zur Übersicht siehe Mayer 2000). Die Kataplexien sind me-

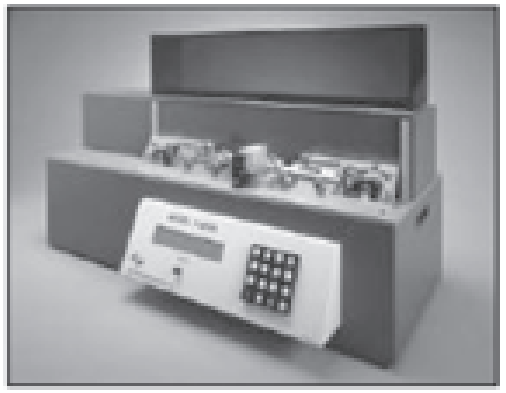

SCIENCE PRODUCTS (SPT) The Full System Concept

Data Acquisition and Data Analysis Systems

Electrodes, Electrode Holders Wires and Glasses

Micropipette Pullers, Microforges and Bevelers

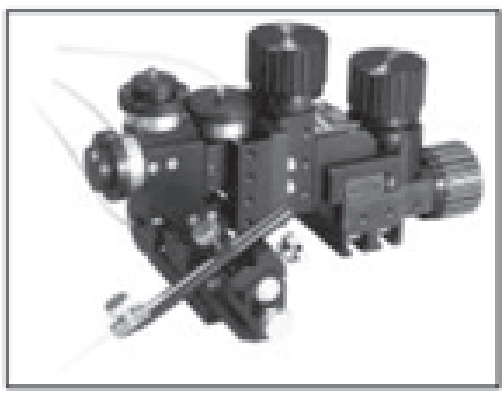

Micromanipulators, Tables and Faraday Cages

Microinjection Systems, Solution Changers

Amplifier, Stimulators and Stimulus Isolators Laboratory Animal Research Equipment Temperature Controllers
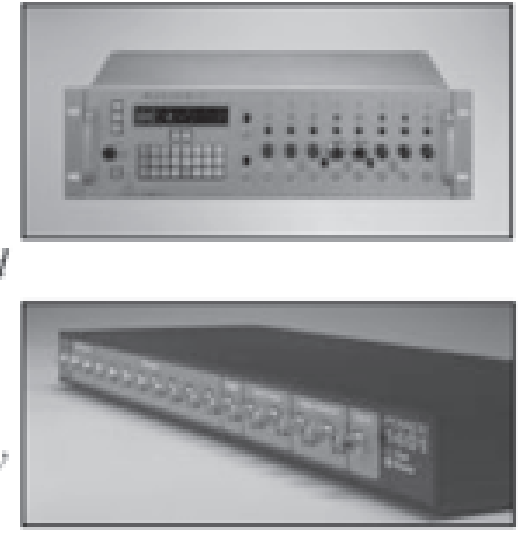

... and more!

Hotheimer Str. 6365719 Hofheim TeL: 06192/ 901396 Fax: 06192/901398 www.science-products.com e-mail: infoescience-products.cam 
dikamentös besser beherrschbar als die Tagesschläfrigkeit, spielen aber meist bzgl. beruflicher oder sozialer Einschränkungen eine geringere Rolle. Einige Patienten können kataplexieauslösende Faktoren unterdrücken.

Die Symptome Tagesschläfrigkeit und automatisches Verhalten lassen sich mittels Stimulanzien behandeln. Die Auswahl der meist amphetamin ähnlichen Präparate hat sich in den letzten Jahren u. a. wegen unzureichender evidenzbasierter Studien stark reduziert. Neben Coffein stellen Modafinil und MAOHemmer die einzigen Präparate dar, die andere Wirkmechanismen haben als Amphetaminderivate. Sowohl antikataplektische als auch stimulierende Medikamente haben Wirkungen auf verschiedene Neurotransmittersysteme (noradrenerg, serotonerg, gabaerg) und verweisen auf die Heterogenität der Symptomursachen.

\section{Ausblick}

Die Ergebnisse der Narkolepsieforschung während der letzten Jahre haben das Interesse der klinischen und präklinischen Forschung an dieser faszinierenden Erkrankung ganz immens gesteigert. Dennoch hat die Entdeckung der Rolle von Orexinen in der Pathophysiologie der Narkolepsie mehr drängende Fragen aufgeworfen, als beantwortet. Die drei zentralen Fragen, die es zu beantworten gilt, lauten: 1.) Ist das erworbene Orexindefizit im Rahmen der humanen Narkolepsie Ausdruck eines Untergangs der orexin-produzierenden Zellen oder stellen diese Zellen die Produktion von Orexinen aus unbekannten Gründen ein? 2.) Wenn es sich um einen Zelluntergang handeln sollte, sind dessen Ursachen primärdegenerativer Natur oder induziert durch exogene Ursachen wie zum Beispiel Infektion, Entzündung oder einen Autoimmunprozess? 3.) Welche genaue Rolle spielt die genetische Suszeptibilität in diesem komplexen pathogenetischen Prozess? Die Beantwortung dieser Fragen könnte zu einer Klärung des Unterschieds zwischen symptomatischen und idiopathischen Narkolepsien, sowie der unterschiedlichen Zeitpunkte der Manifesta-tion narkoleptischer Symptome beitragen.

Darüber hinaus bedarf natürlich auch die genaue Einbindung des Orexinsystems in die seit Jahrezehnten bekannten schlafregulierenden Neurotransmitter- und Neuropeptidsysteme detaillierter Aufklärung und es erscheint ebenso wichtig, die endokrin-metabolischen Aspekte der Narkolepsie genauer zu untersuchen. Auch für die häufigsten komorbiden Störungen müssen diese $\mathrm{Zu}$ sammenhänge geklärt werden. Und schließlich, und dies mag gerade für die betroffenen Patienten allererste Priorität haben, wird zu klären sein, welche Möglichkeiten es gibt, über eine pharmakologische Beeinflussung des Orexinsystems die Therapie der Narkolepsie zu optimieren.

\section{Literatur}

Chemelli, R.M., Willie, J.T., Sinton, C.M., Elmquist, J.K., Scammell, T., Lee. C., Richardson, J.A., Williams, S.C., Xiong, Y., Kisanuki, Y. et al. (1999): Narcolepsy in orexin knockout mice: molecular genetics of sleep regulation. Cell 98(4): 437-451.

Dalal, M.A., Schuld, A., Beitinger, P., Bubendorff, V. und Pollmächer, T. (2002): Neuroendocrine and metabolic aspects of narcolepsy. Somnologie 6: 95-100.

Mayer, G. (2000): Narkolepsie. Berlin: Blackwell Verlag

Mignot, E., Lammers, G.J., Ripley, B. et al. (2002): The role of cerebrospinal fluid hypocretin measurement in the diagnosis of narcolepsy and other hypersomnias. Arch Neurol. 59(10): 15531562.

Schuld, A., Hebebrand, J., Geller, F. und Pollmächer, T. (2001): Increased body-mass index in patients with narcolepsy. The Lancet 355:12741275 .

Eine ausführliche Literaturliste kann bei den Autoren angefordert werden.

\section{Kurzbiographien}

Geert Mayer: 1968-1974 Studium der Medizin in Göttingen, Marburg und Giessen. 1979 Promotion am Hygiene-Institut der Georg-August-Universität zu Göttingen. 1974-1984 Ausbildung zum Facharzt für Neurologie und Psychiatrie. Ab 1986 Oberarzt an der Hephata Klinik SchwalmstadtTreysa, seit 1998 ärztlicher Direktor. 1999 Habilitation an der Universität Göttingen. Wissenschaftlicher Schwerpunkt Schlafmedizin: Narkolepsie, Kleine Levin Syndrom, zirkadiane Schlafstörungen, Parasomnien. Seit 1992 Mitglied des erweiterten Vorstands der Deutschen Gesellschaft für Schlafforschung und Schlafmedizin. Mitherausgeber des wissenschaftlichen Organs der Deutschen Gesellschaft für Schlafforschung und Schlafmedizin „Somnologie“.

Thomas Pollmächer: 1977-84 Studium der Medizin und Alten Geschichte in Freiburg. 1984 Promotion in Medizin, 1998 Habilitation im Fach Psychiatrie. Derzeit Forschungsgruppenleiter und stv. Klinikdirektor am Max Planck Institut für Psychiatrie und Professor für Psychiatrie an der Ludwig Maximilians Universität München. Forschungsschwerpunkte: Psychoneuroimmunologie, Endokrine und immunologische Effekte von Psychopharmaka, Narkolepsie.

\section{Korrespondenzadresse}

PD Dr. med. Geert Mayer

Hephata Klinik

Schimmelpfengstr. 2

D-34613 Schwalmstadt-Treysa

Tel.: ++ 49 (0) 6691182002

Fax: ++ 49 (0) 6691182040

e-mail:geert.mayer@hephata.com

\section{Neueintritte}

Folgende Kolleginnen und Kollegen dürfen wir als Mitglieder der Neurowissenschaftlichen Gesellschaft begrüßen:

Baars, Cordul

(Hannover)

Bergmann, Olaf (Berlin)

Chaimow, Denis

Colla, Dr. Michael

(Tübingen)

Engler, Dr. Gerhard (Berlin)

Faran, Dr. Samuel

Fluegge, Anna Maria

(Hamburg)

Foeller, Dr. Elisabeth

(Tübingen)

Franke, Dr. Heike (Berlin)

Hennig, Matthias H.

Hobom, Dr. Muriel

Juengling, Kay

(La Jolla)

(Leipzig)

(Stirling)

(Göttingen)

(Bochum)

Jungnickel, Dr. Julia

Keipert, Christine

(Hannover)

Kozyryev, Vladyslav

Krajacic, Aleksandra

(Frankfurt)

(Tübingen)

(Bremen)

Leitner, Dr. Stefan

(Seewiesen)

Moeller, Sebastian

(Bremen)

Oreja-Guevara, Dr. Celia (Bochum)

Patrona, Ekaterini (Frankfurt/Main)

Pauli, Prof. Dr. Paul (Würzburg)

Pawlak, Dr. Cornelius (Marburg)

Pietrzik, Prof. Dr. Claus (Mainz)

Pisot, Kristina

Reisch, Adrian

(Hamburg)

(Biberach)

Rybacki, Konrad

(Düsseldorf)

Sams-Dodd, Dr. Frank (Biberach a.d. Riess)

Seelig, A. H. Alexander(Montreal, Quebec)

Sommer, Dr. Bernd (Biberach)

Stemmann, Heiko (Bremen)

Zappe, Anne-Catherin (Gaiberg)

Zimmermann, Dr. Peter (Meckenheim)

Der Mitgliedsstand zum 1. November 2003 beträgt 1.619 Mitglieder. 